\title{
Development of an Energy-Aware Algorithm for Low Power Wireless Sensor Node Powered by Dual Energy Harvesting Sources
}

\author{
Ali Mohammed Abdal-Kadhim, Kok Swee Leong
}

\begin{abstract}
A novel self-powered wireless sensor node is proposed and prototyped to overcome the ambient energy lacking in the dual energy harvesting sources by including a secondary energy storage. Moreover, an energy-aware Event-Priority-Driven Dissemination (EPDD) management algorithm has been developed and implemented to control the WSN integrity and reducing the sensor node power consumption as well. EPDD was developed to manage the sensor node operation and to make the sink station able to detect a missing wireless node within the network, which will guarantee the nodes integrity detection. The evaluations revealed that the EPDD shows a good performance in reducing the node power consumption compared to the data push algorithm, whereby, EPDD node was operating 4 hours more than the data push node on the same power source. Regarding the WSN integrity, the EPDD algorithm outpaced the event trigger algorithm, whereby, the EPDD was easily able to detect a node down within the WSN at the contrary of the event trigger.
\end{abstract}

Keywords : Wireless Sensor Network, Dual energy harvesting sources, EPDD algorithm, Node down detection, WSN integrity.

\section{INTRODUCTION}

$\mathrm{W}_{\mathrm{i}}$ ireless sensor networks WSNs powered by energy harvester devices can provide constant monitoring without the need of batteries and expensive maintenance process, attracting great research interest. Such sensor nodes are able to capture the ambient ubiquitous energy around us like impact and vibration [1], solar and thermal [2], wind energy [3], etc., which is then been recovered into a small but useful amount of electrical energy. This small portion of the electrical signal is then conditioned and regulated with the help of power management circuits to suit the load demand. In light of this, many of researchers were focused on a single source energy harvester like, Rolf et al. proposed a self-powered Internet of Things (IoT) device [4] where it was powered by indoor solar energy harvester for temperature, humidity and light levels mentoring. A wireless gas sensor node powered by RF energy is proposed by Alexander et al. [5]. Pukar presented an indoor power line based magnetic field energy harvester, as a sustainable and maintenance free power supply for self-powered wireless monitoring sensors in

Revised Manuscript Received on July 22, 2019.

Ali Mohammed Abdal-Kadhim, Faculty of Electronic and Computer Engineering, Universiti Teknikal Malaysia Melaka, Melaka, Malaysia. Email: ali.challenger89@yahoo.com

Kok Swee Leong, Faculty of Electronic and Computer Engineering, Universiti Teknikal Malaysia Melaka, Melaka, Malaysia. Email: sweeleong@utem.edu.my

smart home applications. The proposed node harvests energy from the magnetic field induced by a current carrying conductor without electrically contacting the conductor, that are easily available in indoor power line system [6]. Yet, the recovered power by the single source energy harvester is highly unreliable since it is depending on a single ambient energy source. In which it opened the door for other researchers to take a step forward and propose a hybrid/multisource energy harvesting schemes to increase the harvester reliability, like, Jingui et al. where they proposed a wind and solar hybridized energy harvester to develop a self-powered natural disaster wireless monitoring system [7]. Solar energy hybridized with electromagnetic energy along with a Mixed Integer Programming (MIP) method to power and minimize the energy dissipation of sensor nodes for smart grid monitoring applications is presented in [8]. Fang et al. proposed a design, implementation and characterization of a multisource energy harvesting system to drive a sensor node [9]. The multisource energy harvesting module is able to collect ambient energy from the surrounding environment, including wind, solar radiation, and thermal energy.

As aforementioned, the hybrid energy harvesting scheme is proposed to overcome the single harvester drawback (i.e. in case of the ambient energy source is missing), in which the hybrid topology can utilize more than one ambient energy to increase the power supply availability. However, all the proposed hybrid solutions haven't discuss the scenarios in which the ambient energy sources are available at. Where it is expected that the ambient energy sources might not be available at the same time, and/or might both of the ambient energy sources are not available at the same time. Thus, it is prone to have an energy gap due to the lack of both of the energy sources, which will be addressed in the research.

For instance, in an industrial environments and especially in the petroleum outfield, electrical power supplies are strictly prohibited around the gas and oil pipelines area. Thus, it is challenging for the monitoring and alarming systems to be powered and sustained, that would cost the companies more labors to frequently visualize the pipelines areas for avoiding gas leaking, corrosion, violent evil-doings, etc. [10-11]. On the other hand, most of the available ambient energies in such environments are the heat and vibration, which is fortunately can be recovered by the means of hybrid energy harvester to power up the WSN. 
Through the literature, there are several successful tries to utilize energy harvesting in the oil and gas industry, however, still lacking in power source reliability [12], limited to external damages (sabotage activities), and unable to detect leakages and corrosions (self-destruction) [11]. Thus, in this paper, dual source hybrid harvesters will be presented to circumvent the single harvester drawbacks. Add-on, a novel wireless sensor node hardware design will be developed to overcome the energy gap when the ambient energy is not available. Lastly, an energy-aware Event-Priority-Driven Dissemination EPDD management algorithm will be implemented to control the WSN integrity and reducing the sensor node power consumption. Regarding the heat and vibration energy harvesting from an industrial environment, the ambient energy availability might be mismatched according to the scenarios shown in the Fig 1 .

The ambient energy availability is basically time-varying as shown in the Fig. 1 [13-19]. Usually, there would be three different scenarios in accordance with the ambient energy availability. For example, when the machine is off for long time, there will be neither heat nor vibration energy as in the scenario_3. The opposite is true, the machine will be generating a noticeable amount of heat and vibration energy since it operates for long period of time as in the scenario_1. On the other hand, starting the machine from cold-start will generate a vibration energy but no heat energy. At the contrary, when the machine is turned off after a long period of operating, it will stop generates a vibration energy while continuing emitting heat energy to the environment, as it reflected in the scenario_2. Scenarios 1 and 2 are considered manageable by employing dual harvesting sources so it can harvest any ambient energy source available at that time, whereas, scenario 3 is a fatal zone even for the dual source harvester. Thus, a sensor node that is equipped with a single energy harvester and doesn't contains any sort of energy storage/backup will definitely facing a fatal power problem.

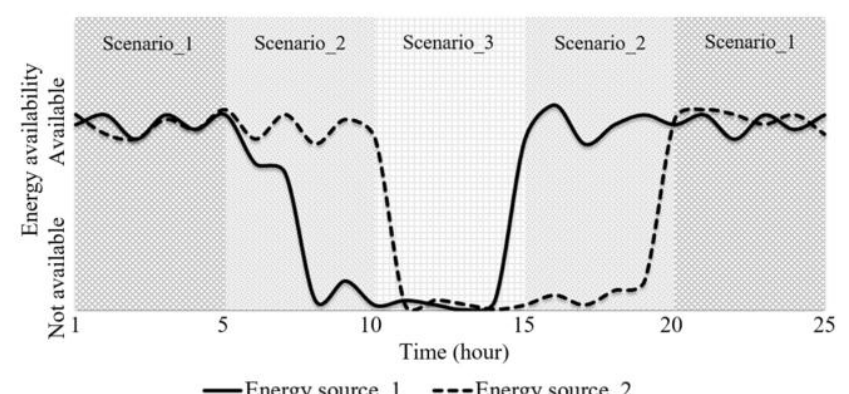

Fig. 1: Ambient energy (heat and vibration) availability scenarios [13-19].

\section{WIRELESS SENSOR NODE HARDWARE/SOFTWARE ARCHITECTURE}

In this research, a fully autonomous wireless sensor node has been designed and prototyped to circumvent the single energy harvester and energy gap issue in two phases. The node hardware architecture design will be implemented in the first phase, and the second phase will describe the node software. The new proposed node hardware architecture block diagram is shown in Fig. 2, in which it comprises two main parts: the dual energy harvesting sources (power supply layer) and the wireless sensor node (load layer). The node power supply is composed of two energy harvesters: piezoelectric transducer for vibration energy harvesting, and thermoelectric generator TEG for heat energy harvesting. A power conditioning circuit based on step-up switching controller MAX1771 was designed to boost up the thermoelectric harvester voltage. It was able to start operating at low voltage of $2 \mathrm{~V}_{\mathrm{DC}}$ harvested by the TEG at temperature gradient of $\Delta \mathrm{T}=50^{\circ} \mathrm{C}$ [20]. The MAX1771 supports an adjustable output thus, a voltage divider of R1 and R2 was connected to its feedback pin to regulate the output to $3 \mathrm{~V} 3$. Then, this voltage would be stored in the $24.5 \mathrm{mF}$ primary energy storage before supplying it to the rest of node components. Fig. 3 illustrates the TEG power conditioning circuit schematic. Since the piezoelectric transducer generates very low magnitude of alternating current $\mathrm{AC}$ with very high voltages, a full-wave bridge rectifier based on schottky diodes with a DC-DC buck converter based on MAX638 were used to condition the power of the piezoelectric. The MAX638 were chosen due to its low operation current $135-180 \mu \mathrm{A}$ which is suitable for the piezoelectric application, high conversion efficiency $85 \%$ and very few passive components are required for its circuit. This DC-DC buck converter also was set to regulate the output to $3 \mathrm{~V} 3$ with the help of the voltage divider R5 and R6. Fig. 3 illustrates the piezoelectric power conditioning circuit schematic. Both of the conditioning circuits were set to regulate the output to $3 \mathrm{~V} 3$ as aforementioned where at this voltage, a minimum power consumption by the MCU can be achieved. At the end, they conserved the energy in the $24.5 \mathrm{mF}$ primary energy storage.

The load layer is consist of Atmel SMART SAM3X flash microcontroller based on the high performance 32-bit ARM Cortex-M3 RISC processor. A temperature sensor LM35 with voltage signal of $10 \mathrm{mV} /{ }^{\circ} \mathrm{C}$ and high speed high sensitivity NPN silicon phototransistor YG1006 to perform flame detection with wavelength of 760 1100nm, and it is able to detect the flame up to $100 \mathrm{~cm}$. A low power operational amplifier used as a comparator for the flame detector phototransistor to interrupt the MCU from the low power mode when an interesting event takes place (fire occurred). Fig. 4 depicting the load layer schematic and how the sensors are connected to the MCU. A $433 \mathrm{MHz}$ RF wireless multichannel half duplex transceiver module (HC-12) is used as the main node transceiver module.

In the proposed node power supply layer, a 500mAh rechargeable LiPo battery is used as a secondary power storage with its own control and protection circuit. This secondary storage will be continuously charging whenever the ambient energy sources are available. Since the wireless transceiver module is the most power hungry module in the sensor node, the secondary power storage will be used only when the node need to transmit a data package out and the ambient energy sources are not available. Moreover, the node MCU is capable of detecting the availability of the two ambient energy sources (heat and vibration) by measuring their voltages via ADCs channels, also it is capable of checking the residual power in the primary energy storage. Fig. 5 
shows the schematic of the secondary storage with the control and protection circuit. From Fig. 5, the control and protection circuit that been used for the LiPo battery is mainly based on a programmable charge current complete constant-current/ constant-voltage linear charger TP4056 for single cell LiPo battery. For protection purpose, a single cell LiPo battery protection DW01A is used to protect the battery from being overcharged, overly discharged (with accuracy of about \pm 50 $\mathrm{mV}$ ) or overcurrent by controlling the low on-resistance dual $\mathrm{N}$-channel enhanced mode power MOSFET FS8205A as it is shown in the Fig. 5.

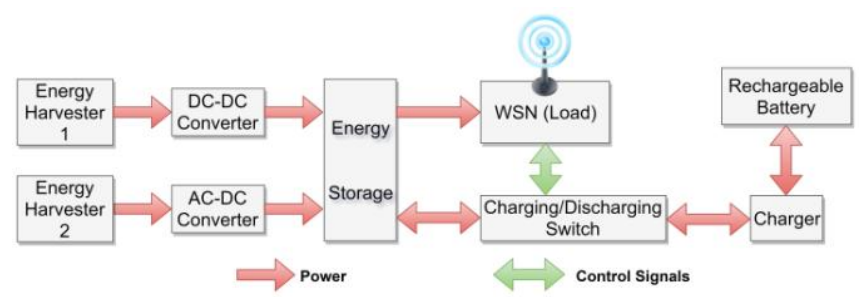

Fig. 2: Proposed wireless sensor node architecture block diagram.

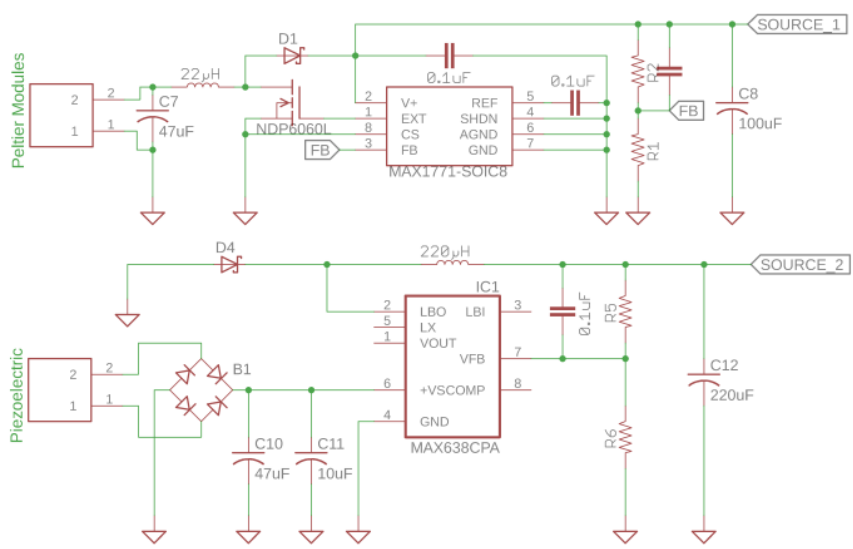

Fig. 3: Proposed sensor node power conditioning circuits' schematic.

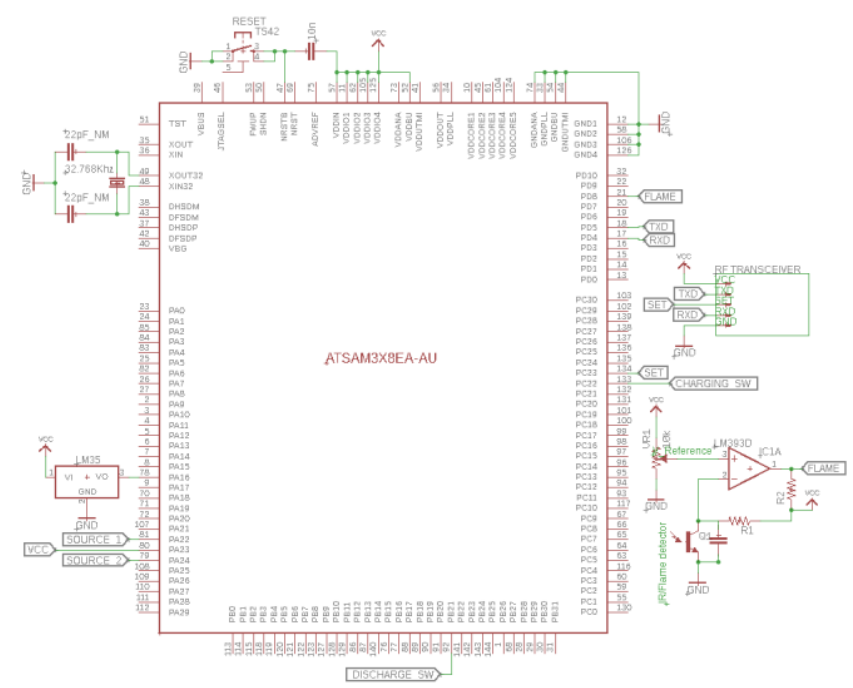

Fig. 4: The load layer schematic of the proposed sensor node.

The SAM3X MCU with the help of the EPDD algorithm will control the LiPo charging and discharging through two switching circuits which are based on general purpose switching transistors BC557 and BC547 as shown in Fig. 5.
The control will be on the power line ( $\mathrm{Vcc}$ ). The MCU will start charging the LiPo whenever the ambient sources are available and use it only when the sources are missing and there is a data packet needed to be transmitted. However, during the normal sensor node operation (i.e. no transmission required) the node will be able to survive with the $24.5 \mathrm{mF}$ primary energy storage. Two NSR0320MW2T1 schottky barrier diodes were used as an ORing diodes (D1 and D2) to isolate and protect the rest of the system in case of a harvester damage or malfunction. The NSR0320MW2T1 schottky barrier is chosen due to its high current handling capability, and low forward voltage performance as shown in Fig. 5. The full-scale prototyping of the proposed wireless sensor node is presented in Fig. 6.

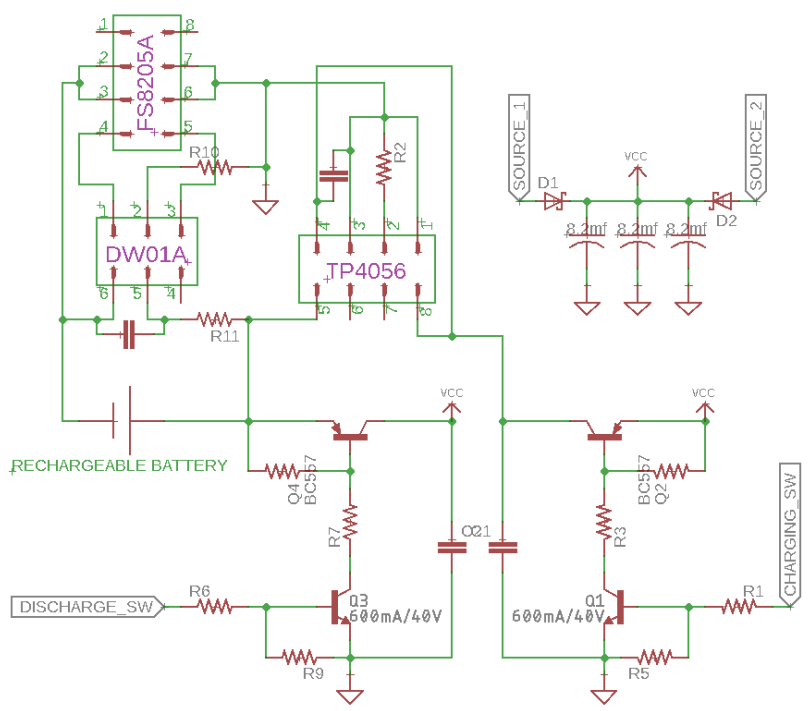

Fig. 5: LiPo battery control and protection circuit schematic.

Regarding the sensor node software design phase, the operating software should be an energy aware to cope with the available energy and should maintain the overall network integrity as well. Also, it will take care of the data reporting strategy, node activity, and resources management. Commonly there are three main data reporting strategies [21] 1) Continuous and periodic reporting (data pushing): in which the sensor node continuously reporting the data to the sink station following a periodic schedule. Data pushing strategy is proactively pushing the data to the network, (and an energy cost incurred) even when the sensed parameter has not significantly changed hence containing little useful information since the previous transmission [21]. Although this strategy will guarantee high level of network integrity, but it will consume very high of power due to the continuous transmission, which make it out of the consideration for energy harvesting powered node. 2) Query driven reporting (data pulling): in this approach the end user instigates data transfer by querying specific data from a specific sensor node in the network. In this strategy the data is pulled out from a passive network in response to queries [22]. The data pulling strategy is very low power consumption, however, 


\section{Development of an Energy-Aware Algorithm for Low Power Wireless Sensor Node Powered by Dual Energy Harvesting Sources}

it is out of the consideration due to the fact it is unable to detect an event and has to manually request the data from the wireless node. 3) Event trigger reporting: in this strategy the sensor node will be intelligent to decide when data should be reported to a sink station. The sensor node that equipped with event trigger reporting will reactively reports the event to the sink station. With locally determining when event occurred that is worth reporting, redundant data transmissions can be minimized. The event trigger approach is also consuming very low amount of power, however, it is out of the consideration due to its low network integrity especially for the energy harvesting applications in which the sink station cannot differentiate between a down node due to the ambient energy missing or no event occurred. Fig. 7 shows conclusions of the data reporting strategies [23].

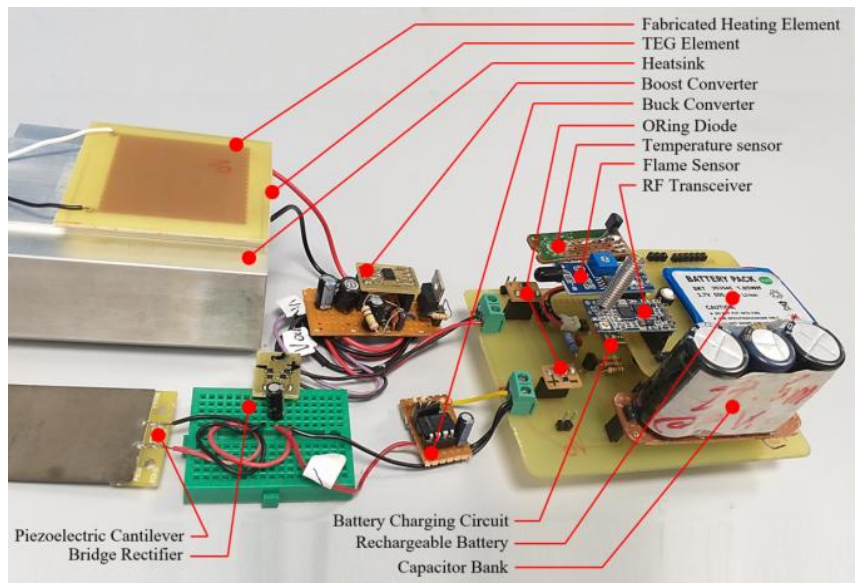

Fig. 6: The prototyping of the proposed WSN_4_DEH.

Therefore, in this research a new improved algorithm called Event-Priority-Driven Dissemination (EPDD) has been developed based on the event trigger reporting strategy to make the sink station capable of differentiating between no event to trigger the sensor node or the node down due to power depletion. The EPDD algorithm that presented in this paper is an improvement of the prior research that presented in [20]. The enhanced EPDD algorithm that presented here will transmit the data package based on the event occurrence, and it will transmit an acknowledgment package only when high priority package achieved, which it will guarantee high network integrity. Fig. 8 shows the proposed enhanced EPDD algorithm.

The first time the EPDD is being launched and directly after it initiate the variables (assuming source 1 and 2 are available, timer $=1$ hour) and the node modules, it will start charging the secondary power storage. Then, it will enter an infinite loop in which it waiting for an event of interest to take place or the timer to be expired. Upon an event of interest takes place, EPDD will directly run a subroutines to check the ambient sources availability, if both of the sources are available the data package (Fig. 9(A)) will be transmitted immediately. If not the EPDD will connect the secondary power storage to the node power network, transmit the data package out and disconnect it again. When the timer is expired and no event of interest occurred, EPDD will increase the package priority level and go into low power again. This algorithm has ten levels of package priority; Low, Medium, and High with representations, 1, 5, and 10 respectively. Whenever high priority level is reached and the ambient sources are available, the node will transmit an acknowledgment package (Fig. 9(B)) immediately, but if the sources are not available it will connect the secondary power storage to the node power network, transmit the acknowledgment package out and disconnect it again. In the EPDD the timer value $s$ is depending on the ambient sources availability as it is clear in the Fig. 8, where the s value will be equal to $(1,2$, or 5 hours) when all the sources are available, only one available, or none of them are available respectively. To recapitulate, the node with the EPDD algorithm will always charging the secondary power storage whenever the ambient sources are available, and will use the secondary power storage whenever the ambient sources are not available and there is a data or acknowledgment package need to be transmitted.

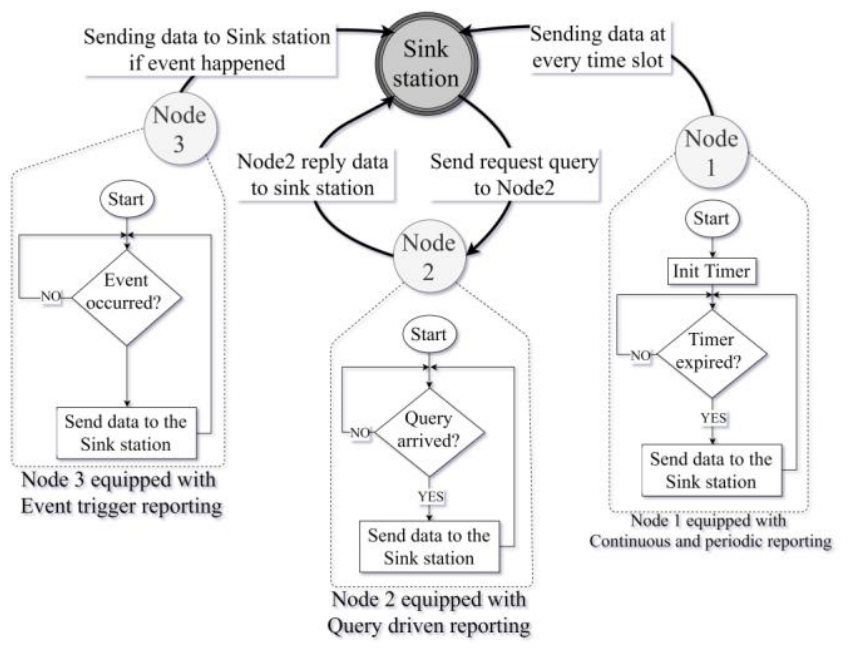

Fig. 7: Conclusions of the data reporting strategies.

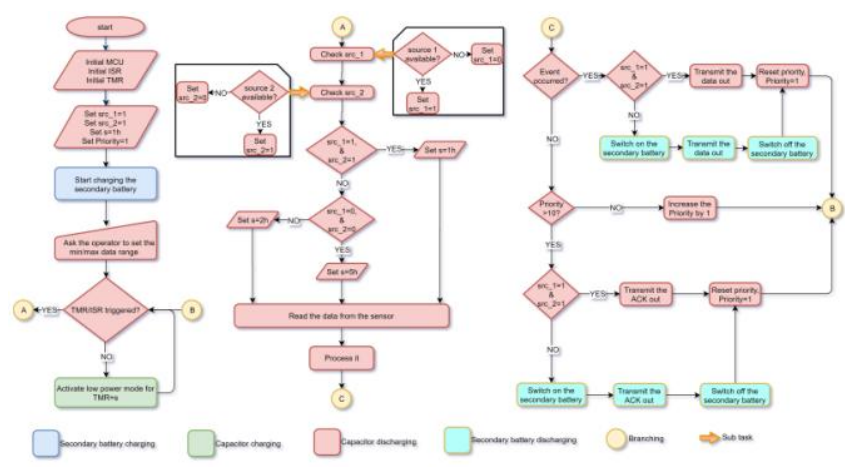

Fig. 8: The proposed Event-Priority-Driven Dissemination EPDD algorithm.

With this technique the power hungry module RF [24] will be always consume power from the secondary power storage only when there is no ambient energy sources. The primary power storage will not be disturb to guarantee a smooth operation of the wireless sensor node.

\section{(A)}

\begin{tabular}{|c|c|c|c|c|c|}
\hline 1 & 2 & 3 & 4 & 5 & 6 \\
\hline Node ID & Packet ID & Source 1 status & Source 2 status & Sensor 1 data & Sensor 2 data \\
\hline
\end{tabular}


(B)

3

Node ID $\quad$ Packet ID $\quad$ Source 1 status Source 2 status

Fig. 9: The proposed EPDD algorithm packages, (A) data package, (B) acknowledgment package.

Utilizing the EPDD algorithm with the sensor node will make the sink station capable of detecting a missing wireless sensor node by cause of power depletion or hardware fault and notify the operator. Fig. 10 illustrates a timing diagram to visually conclude the proposed sensor node hardware and the enhanced EPDD algorithm when the two ambient sources are available, and when they are fluctuating scenarios.

\section{EVALUATION RESULTS AND DISCUSSIONS}

The proposed wireless sensor node hardware that equipped with the new algorithm EPDD was tested and evaluated in a controlled laboratory environment. The experimental setup consists of the wireless sensor node with a sink station. The first evaluation process aspect was to verify that the EPDD algorithm is able to reduce the sensor node power consumption comparing to the data pushing strategy. In this process a $1.85 \mathrm{WH} 500 \mathrm{mAh} \mathrm{Li-ion} \mathrm{battery} \mathrm{used} \mathrm{to} \mathrm{investigate}$ the operating hours comparing to the data pushing strategy. As it is shown in Fig. 11, the data push algorithm is set to send out the data packages at each second, thus it consumes the highest power and it drained out the battery within 4 hours only, which make it inapplicable for energy harvesting powered applications. The data push algorithm however, considered the most reliable strategy and it guarantee WSN integrity. On the other side, the event trigger algorithm is the most power efficient algorithm among the others, however, it is lacking of the node down detection capability (low network integrity). Thus, the event trigger algorithm also is out of the consideration for energy harvesting powered applications due to its low network integrity. Regarding the proposed EPDD algorithm shown in Fig. 11, it takes about 7.7 hours to fully deplete the battery, which is double the data push time and about half of the event trigger time.

Despite the proposed EPDD algorithm is consuming a fair amount of power in which it fully deplete the battery by about 13 hours faster than the event trigger and about 3 hours better than the data push algorithm, it is conserving the node power and maintaining the WSN integrity respectively. Regarding the wireless sensor node down detection, EPDD shows an outstanding success with no major errors compared to the event trigger in verifying a node down. In this evaluation, the same node/sink station hardware shown in Fig. 4 has been utilized in two rounds. In the first round, the node and the sink station were armed with the typical event trigger algorithm shown in Fig. 7 (node 3), and for the second round they were armed with the proposed EPDD algorithm that shown in Fig. 8. Then, they had been deployed in the same environment to test the nodes' integrity performance with the two algorithms at different scenarios. From the evaluation outcomes shown in the Fig. 12, the sink station that armed with the EPDD algorithm performance was obviously outpaces the sink station that armed with the event trigger algorithm. The event trigger sink station was absolutely unable to detect a down node and it was showing a no-event detected while the node was off due to the power lack, Fig. 12 (T8). On the other hand, the sink station with the EPDD was able to detect a missing node very easy, also, it was successfully covered the energy gab when the ambient source was missing.

(A)

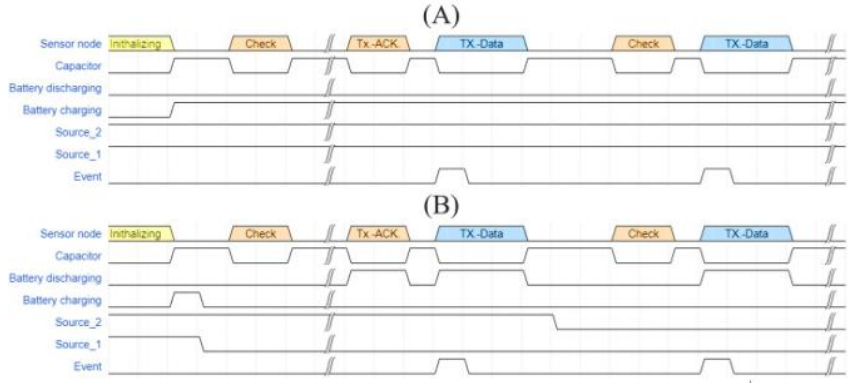

Fig. 10: Timing diagram of the wireless sensor node with EPDD algorithm behavior, (A) ambient sources are available, (B) ambient sources are fluctuating.

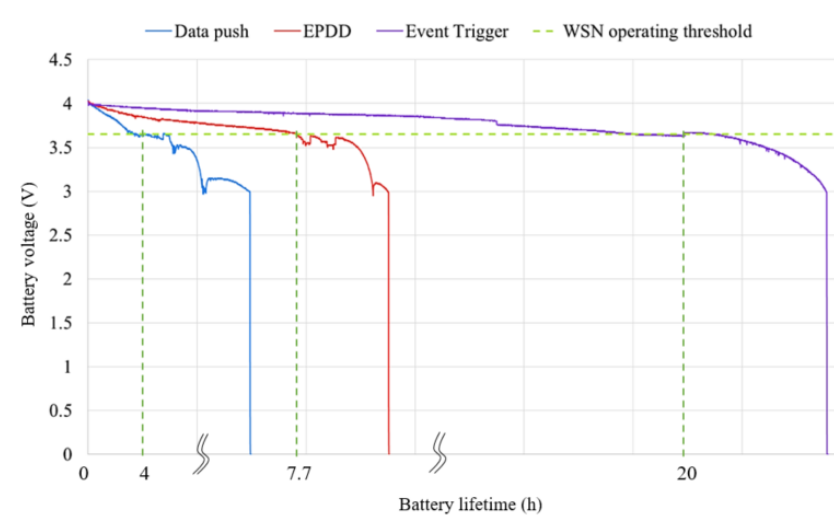

Fig. 11: Battery life time at different data reporting strategies.

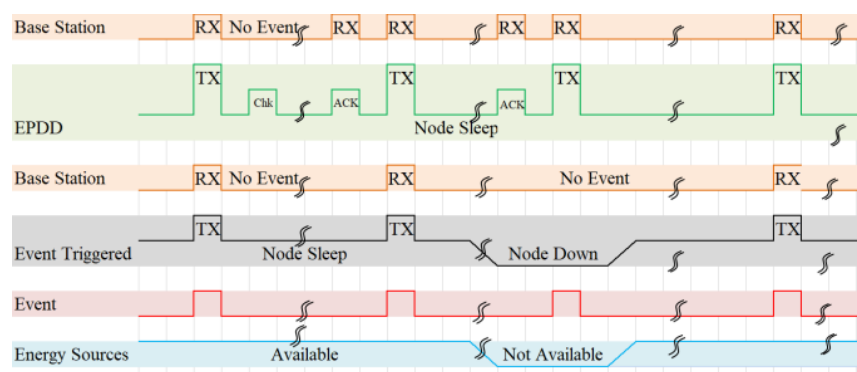

$\begin{array}{lllllllllll}\text { Timing } & \text { T1 } & \text { T2 } & \text { T3 } & \text { T4 } & \text { T5 } & \text { T6 T7 } & \text { T8 } & \text { T9 } & \text { T10 } & \text { T11 }\end{array}$

Fig. 12: Timing diagram of the event trigger vs EPDD algorithms performances comparison.

\section{CONCLUSION}

A novel self-powered wireless sensor node hardware and software were successfully designed and implemented. The sensor node powered by dual energy harvesting sources to solve a single harvester issue. A secondary energy storage is proposed in the hardware to cover the energy gap when there are a lack of the ambient energy sources. Moreover, the proposed Event-Priority-Driven Dissemination EPDD algorithm is not only able to reduce the sensor node power consumption,

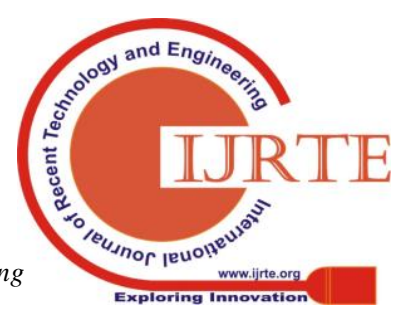




\section{Development of an Energy-Aware Algorithm for Low Power Wireless Sensor Node Powered by Dual Energy Harvesting Sources}

but also tackles the energy gab issue to guarantee high integrity WSN. The evaluation outcomes shows that the EPDD is much better than the data push algorithm by reducing the power consumption, where it was operating 4 hours more than the data push node. Regarding the WSN integrity, the EPDD algorithm outpaced the event trigger algorithm, where it was easily able to detect a node down within the WSN at the contrary of the event trigger.

\section{ACKNOWLEDGMENT}

The authors would like to acknowledge the support of this work by the Malaysian Ministry of Higher Education under the research grant PRGS/1/2016/ TK10/FKEKK- CETRI /02/ T00016, UTeM -Industry Matching GLUAR/ IMPRESSIVE /2017/ FKEKK-CETRI/I00024 and the facility support by the Faculty and ASECs Research Group, CeTRI, UTeM.

\section{REFERENCES}

1. A. M. Abdal-kadhim, S. L. Kok, and N. B. Mohamad, "Application of piezoelectric energy harvesting in powering radio frequency (RF) module", Journal of Telecommunication, Electronic and Computer Engineering, vol. 8, no. 4, pp. 77-79, 2016.

2. S. Ayesha, A. Mehebub, R. M. Tapas, and M. Dipankar, "A pyroelectric generator as a 250 self-powered temperature sensor for sustainable thermal energy harvesting from waste heat and human body heat", Applied Energy, vol. 221, pp. 299-307, 2018.

3. J. C. Hsieh and T. H. Tsai, "An AC-DC Wind Energy Harvesting Circuit with Extended Input-Voltage Range and 95\% Tracking Efficiency", IEEE International Symposium on Circuits and Systems (ISCAS), Florence, Italy, 2018, pp. 1-4.

4. R. A. Kjellby, M. Hamid, and B. B. Lozano, "Self-Powered IoT Device for Indoor Applications", 31st International Conference on VLSI Design (VLSID), Pune, India, 2018, pp. 455-456.

5. A. M. Baranov, et al., "Feasibility of RF energy harvesting for wireless gas sensor nodes", Sensors and Actuators A: Physical, vol. 275, pp. 37-43, 2018.

6. P. Maharjan, M. Salauddin, H. Cho, and J. Y. Park, "An indoor power line based magnetic field energy harvester for self-powered wireless sensors in smart home applications", Applied Energy, vol. 232, pp. 398-408, 2018.

7. J. Qian, and X. Jing, "Wind-driven hybridized triboelectricelectromagnetic nanogenerator and solar cell as a sustainable power unit for self-powered natural disaster monitoring sensor networks", Nano Energy, vol. 52, pp. 78-87, 2018.

8. H. U. Yildiz, V. C. Gungor, and B. Tavli, "A Hybrid Energy Harvesting Framework for Energy Efficiency in Wireless Sensor Networks Based Smart Grid Applications", 17th Annual Mediterranean Ad Hoc Networking Workshop, Capri, Italy, 2018, pp. 1-6.

9. F. Deng, X. Yue, X. Fan, S. Guan, Y. Xu, and J. Chen, "Multisource Energy Harvesting System for a Wireless Sensor Network Node in the Field Environment", IEEE Internet of Things Journal, (Early Access), DOI 10.1109/JIOT.2018.2865431, 2018.

10. M. Y. Aalsalem, et al., "Wireless Sensor Networks in oil and gas industry: Recent advances, taxonomy, requirements, and open challenges", Journal of Network and Computer Applications, (Early Access), DOI: 10.1016/j.jnca.2018.04.004, 2018.

11. C. Dong, S. Li, M. Li, Q. He, D. Xu, X. Li, "Self-Powered Event-Triggered Wireless Sensor Network for Monitoring Sabotage Activities", IEEE Sensors, Orlando, USA, 2016, pp. 1-3.

12. L. J. Klein, M. Ramachandran, T. Kessel, D. Nair, N. Hinds, H. F. Hamann, N. E. Sosa, "Wireless sensor networks for fugitive methane emissions monitoring in oil and gas industry", IEEE International Congress on Internet of Things, San Francisco, CA, USA, 2018, pp.41-48.

13. A. Roberts, R. Brooks, P. Shipway, "Internal combustion engine cold-start efficiency: A review of the problem, causes and potential solutions", Energy Conversion and Management, vol. 82, pp. 327-350, 2014.

14. R. Mudasar, M. Kim, "Experimental study of power generation utilizing human excreta", Energy Conversion and Management, vol. 147, pp. 86-99, 2017.
15. D. Spirjakin, A. M. Baranov, A. Somov, V. Sleptsov, "Investigation of heating profiles and optimization of power consumption of gas sensors for wireless sensor networks", Sensors and Actuators A: Physical, vol. 247, pp. 247-253, 2016.

16. R. Liu, M. Wei, H. Yang, "Cold start control strategy for a two-stroke spark ignition diesel-fuelled engine with air-assisted direct injection", Applied Thermal Engineering, vol. 108, pp. 414-426, 2016.

17. R. Cipollone, D. Battista, M. Mauriello, "Effects of oil warm up acceleration on the fuel consumption of reciprocating internal combustion engines", 70th Conference of the ATI Engineering Association, Roma, 2015, pp. 1-8.

18. T. D. Tan, L. M. Ha, and N. T. Anh, "A Real-time Vibration Monitoring for Vehicle Based on 3-DOF MEMS Accelerometer", Proceedings of International Conference on Computational Intelligence and Vehicular System (CIVS2010), Cau Giay,Ha Noi,Viet Nam, 2010, pp. 1-5.

19. S. W. Hanly. (2016). Shock \& Vibration Testing Overview [Online]. Available https://info.mide.com/data-loggers/shock-vibration-testingoverview-ebook.

20. A. M. Abdal-kadhim, S. L. Kok, and N. B. Mohamad, "Application of Thermal Energy Harvesting in Powering WSN Node with Event-Priority-Driven Dissemination Algorithm for IoT Applications", Journal of Engineering Science and Technology, vol. 13, no. 8, pp. 2569-2586, 2018

21. G. V. Merrett, "Energy-and Information-Managed Wireless Sensor Networks: Modelling and simulation", Ph.D. dissertation, School of electronic and computer science, University of Southampton, October 2008

22. A. W. Khan, J. I. Bangash, A. Ahmed, A. H. Abdullah, "QDVGDD Query-Driven Virtual Grid based Data Dissemination for wireless sensor networks using single mobile sink", Wireless Networks, pp. 1-13. DOI.org/10.1007/s11276-017-1552-8, 2017.

23. A. I. Saleh, K. M. Abo-Al-Ez, A. A. Abdullah, "A Multi-Aware Query Driven (MAQD) routing protocol for mobile wireless sensor networks based on neuro-fuzzy inference", Journal of Network and Computer Applications, vol. 88, pp. 72-98, 2017.

24. A. Potsch, A. Berger, A. Springer, "Efficient Analysis of Power Consumption Behaviour of Embedded Wireless IoT Systems", IEEE International Instrumentation and Measurement Technology Conference (I2MTC), Turin, Italy, 2017, pp. 1-6.

\section{AUTHORS PROFILE}

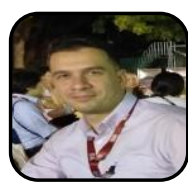

Ali Mohammed Abdal-Kadhim received his M Eng. degree in electronic and computer engineering from the UTeM, Malaysia in 2015, exploring embedded system design and robotics. He is currently pursuing the $\mathrm{Ph} . \mathrm{D}$. degree at the same university. His research interest includes embedded system and WSN design for IoT applications based hybrid energy harvesting. He has involved in several projects related to WSN based energy harvesting for telemetry monitoring also authored few papers related to WSNs and energy harvesting.

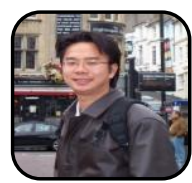

Kok Swee Leong was awarded Ph.D in Electronics and Electrical Engineering from the Southampton University, UK in 2010, exploring thick-film technologies for fabricating piezoelectric energy harvesting devices. Dr. Kok has been a lecturer in UTeM since 2003 after completing his MSc degree in Electrical, Electronic and System Engineering from UKM, researching on optical planar waveguide fabrication and characterization. $\mathrm{He}$ is currently an Associate Professor and also research leader of Advanced Sensors and Embedded Control Systems (ASECs) Research Group under the Center of Telecommunication and Innovation (CeTRI), UTeM. He have been author and co-author for more than 50 publications and reviewing IEEE, IOP, Elsevier and other Scopus indexed journals. His research interest is related to energy harvesting system and applications, MEMS, and thick-film devices fabrication technologies for the application of sensing, actuating and self-powering for wireless sensor network. 